\title{
EFEITO DA ATMOSFERA MODIFICADA E DA REFRIGERAÇÃO NA CONSERVAÇÃO PÓS-COLHEITA DE MANGA ESPADA VERMELHA ${ }^{1}$
}

\author{
LUCIANA BITTENCOURT PFAFFENBACH'르. JOSALBA VIDIGAL DE CASTRO², CÁSSIA REGINA LIMONTA \\ CARVALHO $^{3}$, CARLOS JORGE ROSSETTO ${ }^{4}$
}

\begin{abstract}
RESUMO - Este trabalho visou avaliar o comportamento pós-colheita da variedade de manga Espada Vermelha em refrigeração e o potencial da tecnologia de atmosfera modificada na conservação pós-colheita. A atmosfera modificada foi conseguida através do uso de PVC (6 $\mu \mathrm{m}), \mathrm{PEBD}(25 \mu \mathrm{m})$, PEBD $(25 \mu \mathrm{m})$ com sachê absorvedor de etileno de permanganato de potássio, filme de permeabilidade seletiva aditivado com absorvedor de etileno (Conservax) e controle (sem filme plástico). Os frutos foram mantidos a $12^{\circ} \mathrm{C} \mathrm{e} 90 \%$ UR. A avaliação da qualidade foi feita semanalmente logo após a saída dos frutos da refrigeração e após a sua permanência por 4 dias, em temperatura ambiente. Foram feitas determinações de perda de peso individual dos frutos, evolução da cor da casca e da polpa, taxa de firmeza dos frutos, ocorrência de manchas deteriorativas, pH da polpa, teores de sólidos solúveis ( ${ }^{\circ}$ Brix $)$, porcentagem de acidez (\% de ácido cítrico) e cálculo da relação ${ }^{\circ}$ Brix/acidez. PEBD+sachê influenciou positivamente a manutenção da qualidade e o Conservax prejudicou a maturação dos frutos de manga.
\end{abstract}

Termos para indexação: manga, pós-colheita, embalagem, qualidade do fruto.

\section{THE EFFECT OF MODIFIED ATMOSPHERE AND REFRIGERATION ON POST-HARVEST OF MANGO RED ESPADA}

\begin{abstract}
The present research was aimed to evaluate the post-harvest behavior of mango Red Espada in refrigeration and the potencial of the modified atmosphere technology, tending to permit a best post-harvest conservation of mangoes. The modified atmosphere was obtained using PVC $(6 \mu \mathrm{m}), \operatorname{PEBD}(25 \mu \mathrm{m}), \operatorname{PEBD}(25 \mu \mathrm{m})$ with ethylene absorber sachet, permeable selective film with ethylene absorber in its structure (Conservax) and control (without film). The fruits were maintained at $12^{\circ} \mathrm{C}$ and $90 \% \mathrm{RH}$. The quality valuation occurred weekly after refrigeration and after permanence of fruits at ambient air temperature. Parameters as fruit weight loss, peel and fresh color, firmness, decay incidence, pH, total soluble solids, titratable acidity and their ratios were evaluated. PEBD+sachet affected positively the quality maintainence and Conservax harmed the maturity of mangos.

Index terms: mango, post-harvest, package, fruit quality.
\end{abstract}

\section{INTRODUÇÃo}

A produção de manga no Brasil apresenta grande potencial de crescimento para exportação e mercado interno, sendo que no ano de 2000 a produção brasileira foi de 969 mil toneladas (Agrianual 2002) e as exportações em 2002 atingiram US\$ 50 milhões e 103 mil toneladas (Instituto Brasileiro de Frutas, 2003), conferindo à fruta o primeiro lugar entre as exportadas em valor e o segundo em peso. A variedade IAC 103 Espada Vermelha pode ser usada para a produção precoce de frutos, é produtiva, apresenta moderado teor de fibra e bom sabor (Rossetto et al., 2000). A vida pós-colheita da manga é limitada pela deterioração fisiológica causada pelo excessivo amadurecimento da fruta e pelo desenvolvimento de patógenos que ocasionam podridões. Além disso, a perda de água pelos frutos pode atingir níveis que causam enrugamento e murchamento das mangas e que comprometem o aspecto visual e reduzem seu valor comercial. Segundo Jerônimo \& Kanesiro (2000), o emprego da refrigeração prolonga o período de conservação dos frutos e o uso de atmosfera modificada durante o armazenamento pode reduzir os danos ocasionados pela respiração e pela transpiração, como perda de massa e mudança na aparência. A temperatura de refrigeração recomendada para mangas é de $12^{\circ} \mathrm{C}$ (Alves et al., 1998). Atmosfera modificada pode ser resumida como presença de uma barreira artificial - como embalagem de filme plástico - à difusão de gases em torno do produto, que resulta em redução do nível de $\mathrm{O}_{2}$, aumento do nível de $\mathrm{CO}_{2}$, alteração na concentração de etileno e vapor d'água e alterações em outros compostos voláteis (Lana \& Finger, 2000). No acondicionamento de frutas devem ser aplicadas embalagens ativas, principalmente com absorvedores de etileno, que é um produto indesejável do próprio metabolismo da fruta, pois funciona como acelerador da maturação (Sarantópoulos et al., 1996). Assim, o objetivo deste trabalho foi avaliar as características de qualidade e conservação de mangas em refrigeração e emprego de diferentes embalagens, visando permitir uma melhor conservação pós-colheita da manga in natura, facilitando sua comercialização nos mercados interno e externo.

\section{MATERIALE MÉTODOS}

O experimento foi instalado em novembro de 2001 no laboratório de pós-colheita do Instituto Agronômico (IAC/APTA), em Campinas/SP, com mangas da variedade precoce IAC 103 Espada Vermelha, produzidas em pomar experimental no Pólo Regional de Desenvolvimento Tecnológico dos Agronegócios do Noroeste Paulista, da Agência Paulista de Tecnologia dos Agronegócios (APTA) em Votuporanga, SP. O plantio das mudas foi realizado em agosto de 1995, usando como porta-enxerto a variedade Manila. O espaçamento utilizado foi de $9 \mathrm{~m}$ entre linhas e $8 \mathrm{~m}$ entre plantas nas linhas e os tratos culturais seguiram as técnicas normalmente recomendadas para a cultura na região. Os frutos de 3 plantas foram colhidos manualmente, com a coloração da casca em quantidades iguais de verde e vermelho e selecionados uniformemente quanto à massa, estádio de maturação, ausência de defeitos e de doenças. As mangas estavam firmes e internamente com coloração amarelo claro. O transporte foi feito no mesmo dia para Campinas/ SP, em 4 caixas plásticas de colheita, contendo aproximadamente 60 frutos, cobertas com sombrite, na carroceria de uma caminhonete. No dia seguinte, no laboratório, os frutos foram pesados, embalados, acondicionados e refrigerados. As embalagens utilizadas foram: filme de permeabilidade seletiva aditivado com absorvedor de etileno Conservax, da marca PROBAG; PVC com espessura de $6 \mu \mathrm{m}$; polietileno de baixa densidade (PEBD) com espessura de $25 \mu \mathrm{m}$ de parede simples; polietileno de baixa densidade (PEBD) com espessura de $25 \mu \mathrm{m}$ de pare-

\footnotetext{
(Trabalho 040/2003). Recebido: 14/03/2003. Aceito para publicação: 01/10/2003.

2 Eng. Agr., Ms., Instituto Agronômico (IAC/APTA). CP 28, 13001-970 - Campinas/SP. Fone 0XX19 3241-5188 - R.339 - luciana.luciana@uol.com.br

${ }_{3}^{3}$ PqC VI, Dra., Centro de Pesquisa e Desenvolvimento de Ecofisiologia e Biofísica, IAC/APTA - Campinas/SP. Fone 0XX193241-5188 - R.339 - josalba@iac.sp.gov.br

${ }^{4}$ PqC IV, Ms., Centro de Pesquisa e Desenvolvimento de Recursos Genéticos Vegetais, IAC/APTA - Campinas/SP. Fone 0XX19 $3241-5188$ - R.310 climonta@iac.sp.gov.br.

${ }_{5}^{5}$ PqC VI, Dr., Polo Regional de Desenvolvimento Tecnológico dos Agronegócios do Noroeste Paulista, APTA, CP 61, 15500-000 - Votuporanga/SP. Fone 0XX17 3406-9555 - polonoroestepaulista@apta.sp.gov.br.
} 
TABELA 1 - Perda de massa (\%) de mangas Espada Vermelha, em diferentes embalagens durante armazenamento refrigerado e após transferência e permanência por 4 dias em temperatura ambiente.

\begin{tabular}{|c|c|c|c|c|}
\hline \multicolumn{5}{|c|}{ Tempo de armazenamento em refrigeração (dias) } \\
\hline & 7 & 14 & 21 & 28 \\
\hline Controle & $1,06^{2} \mathrm{a}^{1}$ & $1,84 \mathrm{a}$ & $2,78 \mathrm{a}$ & $3,36 \mathrm{a}$ \\
\hline PEBD & $0,34 \mathrm{~b}$ & $0,51 \mathrm{c}$ & $0,62 \mathrm{~cd}$ & $0,78 \mathrm{~d}$ \\
\hline PEBD+sachê & $0,48 \mathrm{~b}$ & $0,94 \mathrm{~b}$ & $0,85 \mathrm{bc}$ & $0,73 \mathrm{~d}$ \\
\hline PVC & $0,50 \mathrm{~b}$ & $0,90 \mathrm{~b}$ & $1,19 \mathrm{~b}$ & $1,82 \mathrm{~b}$ \\
\hline Conservax & $0,44 \mathrm{~b}$ & $0,90 \mathrm{~b}$ & $0,51 \mathrm{~d}$ & $1,17 \mathrm{c}$ \\
\hline CV 9,83\% & & & & \\
\hline \multicolumn{5}{|c|}{ Tempo de armazenamento em refrigeração (dias) + 4 dias em ambiente } \\
\hline & 7 & 14 & 21 & 28 \\
\hline Controle & $3,53 \mathrm{a}$ & $5,33 \mathrm{a}$ & $6,63 \mathrm{a}$ & - \\
\hline PEBD & $3,03 \mathrm{a}$ & $4,26 \mathrm{bc}$ & $3,47 \mathrm{c}$ & - \\
\hline PEBD+sachê & $3,36 \mathrm{a}$ & $4,38 \mathrm{abc}$ & $4,00 \mathrm{bc}$ & - \\
\hline PVC & $3,53 \mathrm{a}$ & $5,20 \mathrm{ab}$ & $4,91 \mathrm{~b}$ & - \\
\hline Conservax & $3,18 \mathrm{a}$ & $3,89 \mathrm{c}$ & $4,41 \mathrm{bc}$ & - \\
\hline
\end{tabular}

CV $6,03 \%$

${ }^{1}$ Médias seguidas pela mesma letra na coluna não diferem entre si pelo teste Tukey a 5\%.

${ }^{2}$ Para análise estatística os dados foram transformados em $\operatorname{arcsen} \sqrt{x / 100}$

de simples com sachê de permanganato de potássio (absorvedor de etileno) - 4,7g de sachê/kg de fruto e controle (sem filme plástico). Depois de embaladas, em grupos de 4 frutos, as mangas foram acondicionadas em caixa de papelão tipo exportação. As mangas foram mantidas em câmara fria à temperatura de $12^{\circ} \mathrm{Ce} 90 \%$ de UR. Um grupo de 4 frutos por tratamento era transferido semanalmente para a temperatura ambiente $\left(24^{\circ} \mathrm{C}, 75-80 \% \mathrm{UR}\right)$, quando eram retiradas as embalagens e os frutos assim permaneciam até o amadurecimento (4 dias), visando simular o período de comercialização. As mangas foram avaliadas após a colheita, na retirada da câmara de refrigeração (após 7, 14, 21 e 28 dias) e após a permanência em temperatura ambiente por 4 dias. As características avaliadas foram: cor da casca, atribuindo-se valores de 1 a 5 , sendo 1-verde, 2-mais verde que vermelho, 3-quantidades iguais de verde e vermelho, 4-quantidades maiores de vermelho que verde e 5-vermelho (Medlicott et al., 1990); cor da polpa, através de escala de notas de 1 a 5 , correspondendo a 1-polpa creme claro, 2-creme, 3-amarelo claro, 4-amarelo alaranjado e 5-alaranjado intenso (Medlicott et al., 1990 e EMEX, 1998); firmeza, de acordo com Polderdijk et al. (2000), significando 1muito firme, 2-firme, 3-moderadamente mole, 4-mole e 5-muito mole e presença de manchas deteriorativas, atribuindo-se valores de 1 a 5 (Sampaio \& Barbin, 1981), sendo 1-fruto sadio, 2-fruto com pequenas manchas (1 a $2 \mathrm{~mm}$ ) e em pequena quantidade, 3-fruto com pequenas manchas, em maior intensidade, ou poucas manchas maiores, 4-fruto com $1 / 3$ ou mais da sua superfície tomada por manchas e 5 -fruto com $2 /$ 3 ou mais de sua superfície tomado por manchas, apodrecido. Avaliouse também a perda de massa individual dos frutos. Na polpa determinouse o $\mathrm{pH}$, teores de sólidos solúveis $\left({ }^{\circ} \mathrm{Brix}\right)$, porcentagem de acidez e cálculo da relação ${ }^{\circ}$ Brix/acidez, de acordo com Carvalho et al. (1990). Foi feita análise de variância dos dados e as médias comparadas pelo teste de Tukey a $5 \%$ de probabilidade.

\section{RESULTADOS E DISCUSSÃO}

A maior perda de massa foi observada no tratamento controle, seguida pelo tratamento onde os frutos foram embalados em PVC. No tratamento controle essa perda foi aproximadamente o dobro da observada nos frutos embalados em PVC, durante todo o período de armazenamento em refrigeração (Tabela 1). No $21^{\circ}$ dia de refrigeração, as perdas de massa foram $2,8 \%$ e 1,2\%, para o controle e o PVC, respectivamente. Jerônimo \& Kanesiro (2000) encontraram este mesmo valor para o tratamento com PVC e valor maior para o controle $(6,5 \%)$, em mangas Palmer armazenadas a $13^{\circ} \mathrm{C}$ por 20 dias. A partir do $14^{\circ}$ dia, os tratamentos com PEBD, o PEBD+sachê e o Conservax foram aqueles que apresentaram menor perda de massa pelos frutos, tanto nos refrigerados como naqueles transferidos para a temperatura ambiente.

No $28^{\circ}$ dia os frutos retirados da refrigeração não foram mantidos em temperatura ambiente, pois já se encontravam no ponto ótimo para consumo (maduros).

Não houve efeito do fator embalagem na evolução da cor da casca durante o armazenamento sob refrigeração (Tabela 2). Jerônimo (2000) também observou que o uso de sachê ou filme plástico fabricado com permanganato de potássio não mostrou diferenças na evolução da coloração da casca de mangas Palmer. Houve, na realidade, evolução da cor em relação ao tempo de armazenamento, estando as mangas, no $7^{\circ}$ dia, com quantidades iguais de verde e vermelho e no $28^{\circ}$ dia, com quantidades maiores de vermelho que verde. Na permanência dos frutos em temperatura ambiente, $o$ Conservax foi a embalagem mais efetiva para o menor desenvolvimento da coloração vermelha da casca dos frutos (Tabela 2), porém os frutos não amadureceram normalmente e a casca ficou com coloração escurecida.

Em refrigeração não houve efeito de embalagem para cor da polpa, conforme se constata na Tabela 2. Verificou-se diferença estatística em relação ao tempo de armazenamento, sendo que os frutos atingiram coloração entre amarelo claro e amarelo alaranjado no $7^{\circ}$ dia e amarelo alaranjado no $28^{\circ}$ dia. Quando transferidos e mantidos em condições ambiente, os frutos do controle e do tratamento com PVC desenvolveram mais rapidamente a cor da polpa (alaranjado intenso) que os do PEBD+sachê (amarelo alaranjado) e principalmente quando comparados aos frutos embalados com o Conservax (Tabela 2).

Na Tabela 3 constata-se que em refrigeração, houve tendência de aumento na presença de manchas deteriorativas nos frutos de todos os tratamentos em relação ao tempo de armazenamento. Entretanto, as diferenças estatísticas só foram observadas a partir dos 21 dias, onde o controle se destacou por ter desenvolvido maior quantidade de lesões que os outros tratamentos, principalmente em relação ao PEBD+sachê e o Conservax, aos 28 dias (Tabela 3). Após a permanência em temperatura ambiente, apenas os frutos armazenados por até 14 dias em refrigeração, apresentaram-se em condições de comercialização e consumo, exceto os do tratamento PVC, no qual as mangas estavam com restrições para comércio. Após 21 dias em refrigeração, os frutos de todos os tratamentos, exceto os embalados em Conservax, quando transferidos para temperatura ambiente, apresentavam grande desenvolvimento de manchas deteriorativas (Tabela 3). O tratamento onde os frutos foram embalados em Conservax, embora tenha propiciado menor desenvolvimento de manchas deteriorativas, conferiu aparência escurecida aos frutos, interna e externamente. $\mathrm{O}$ escurecimento dos tecidos ocorre pelo fato dos níveis de $\mathrm{O}_{2}$ e $\mathrm{CO}_{2}$ alcançados no interior da embalagem terem sido tóxicos para os frutos, de acordo com Lana \& Finger (2000). 
TABELA 2 - Coloração da casca e polpa de mangas Espada Vermelha, em diferentes embalagens, durante armazenamento refrigerado e após transferência e permanência por 4 dias em temperatura ambiente.

\begin{tabular}{|c|c|c|c|c|c|c|c|}
\hline & \multicolumn{4}{|c|}{ Cor da casca ${ }^{1}$ (refrigeração) } & \multicolumn{3}{|c|}{ Cor da casca $^{1}$ (T. ambiente) } \\
\hline Embalagens & 7 & 14 & 21 & 28 & $7+4$ & $14+4$ & $21+4$ \\
\hline Controle & $3,25^{1} \mathrm{a}$ & $3,25 \mathrm{a}$ & $4,00 \mathrm{a}$ & $4,00 \mathrm{a}$ & $5,00 \mathrm{a}$ & $5,00 \mathrm{a}$ & $5,00 \mathrm{a}$ \\
\hline PEBD & $2,75 \mathrm{a}$ & $3,50 \mathrm{a}$ & $3,50 \mathrm{a}$ & $4,00 \mathrm{a}$ & $5,00 \mathrm{a}$ & $5,00 \mathrm{a}$ & $5,00 \mathrm{a}$ \\
\hline PEBD + sachê & $2,50 \mathrm{a}$ & $3,75 \mathrm{a}$ & $4,00 \mathrm{a}$ & $4,00 \mathrm{a}$ & $4,00 \mathrm{~b}$ & $4,50 \mathrm{a}$ & $5,00 \mathrm{a}$ \\
\hline PVC & $2,75 \mathrm{a}$ & $3,25 \mathrm{a}$ & $3,50 \mathrm{a}$ & $3,75 \mathrm{a}$ & $5,00 \mathrm{a}$ & $5,00 \mathrm{a}$ & $5,00 \mathrm{a}$ \\
\hline Conservax & $2,75 \mathrm{a}$ & $3,00 \mathrm{a}$ & $3,25 \mathrm{a}$ & $3,25 \mathrm{a}$ & $3,25 \mathrm{c}$ & $3,25 \mathrm{~b}$ & $3,25 \mathrm{~b}$ \\
\hline $\mathrm{CV} \%$ & \multicolumn{4}{|c|}{20,62} & \multicolumn{3}{|c|}{5,91} \\
\hline & \multicolumn{4}{|c|}{ Cor da polpa $^{2}$ (refrigeração) } & \multicolumn{3}{|c|}{ Cor da polpa $^{2}$ (T. ambiente $)$} \\
\hline Embalagens & 7 & 14 & 21 & 28 & $7+4$ & $14+4$ & $21+4$ \\
\hline Controle & $3,25 \mathrm{a}$ & $4,00 \mathrm{a}$ & $4,00 \mathrm{a}$ & $4,00 \mathrm{a}$ & $5,00 \mathrm{a}$ & $5,00 \mathrm{a}$ & $5,00 \mathrm{a}$ \\
\hline PEBD & $3,50 \mathrm{a}$ & $3,75 \mathrm{a}$ & $4,00 \mathrm{a}$ & $4,00 \mathrm{a}$ & $4,75 \mathrm{a}$ & $5,00 \mathrm{a}$ & $5,00 \mathrm{a}$ \\
\hline PEBD + sachê & $3,50 \mathrm{a}$ & $3,75 \mathrm{a}$ & $4,00 \mathrm{a}$ & $4,00 \mathrm{a}$ & $4,50 \mathrm{ab}$ & $4,50 \mathrm{ab}$ & $5,00 \mathrm{a}$ \\
\hline PVC & $3,50 \mathrm{a}$ & $3,50 \mathrm{a}$ & $3,75 \mathrm{a}$ & $3,75 \mathrm{a}$ & $4,75 \mathrm{a}$ & $5,00 \mathrm{a}$ & $5,00 \mathrm{a}$ \\
\hline Conservax & $3,50 \mathrm{a}$ & $3,75 \mathrm{a}$ & $3,75 \mathrm{a}$ & $3,75 \mathrm{a}$ & $4,00 \mathrm{~b}$ & $4,00 \mathrm{~b}$ & $4,00 \mathrm{~b}$ \\
\hline $\mathrm{CV}$ & \multicolumn{4}{|c|}{$13,33 \%$} & \multicolumn{3}{|c|}{$5,93 \%$} \\
\hline
\end{tabular}

Para análise estatística os dados foram transformados em $\sqrt{x}$.

Médias seguidas pela mesma letra na coluna não diferem entre si pelo teste Tukey a $5 \%$.

${ }^{1}$ Coloração da casca: 1-verde, 2-mais verde que vermelho, 3-quantidades iguais de verde e vermelho, 4-quantidades maiores de vermelho que verde e 5-vermelho.

${ }^{2}$ Coloração da polpa: 1-creme claro, 2-creme, 3-amarelo claro, 4-amarelo alaranjado e 5-alaranjado intenso.

TABELA 3 - Desenvolvimento de manchas e evolução da perda de firmeza de mangas Espada Vermelha, em diferentes embalagens, durante armazenamento refrigerado e após transferência e permanência em temperatura ambiente

\begin{tabular}{|c|c|c|c|c|c|c|c|}
\hline \multirow{2}{*}{ Embalagens } & \multicolumn{4}{|c|}{ Refrigeração } & \multicolumn{3}{|c|}{ Temperatura a mbiente } \\
\hline & 7 dias & 14 dias & 21 dias & 28 dias & $7+4$ dias & $14+4$ dias & $21+4$ dias \\
\hline & \multicolumn{7}{|c|}{ Desenvolvimento de manchas ${ }^{1}$} \\
\hline Controle & $1,00 \mathrm{a}$ & $1,50 \mathrm{a}$ & $3,00 \mathrm{a}$ & $4,75 \mathrm{a}$ & $1,50 \mathrm{~b}$ & $2,25 \mathrm{a}$ & $4,00 \mathrm{a}$ \\
\hline PEBD & $1,00 \mathrm{a}$ & $1,50 \mathrm{a}$ & $1,50 \mathrm{~b}$ & $2,75 \mathrm{bc}$ & $2,25 \mathrm{ab}$ & $2,75 \mathrm{a}$ & $4,25 \mathrm{a}$ \\
\hline PEBD + sachê & $1,00 \mathrm{a}$ & $1,00 \mathrm{a}$ & $2,00 \mathrm{ab}$ & $2,00 \mathrm{~cd}$ & $1,75 \mathrm{ab}$ & $2,75 \mathrm{a}$ & $3,75 \mathrm{a}$ \\
\hline PVC & $1,00 \mathrm{a}$ & $1,75 \mathrm{a}$ & $1,75 \mathrm{~b}$ & $3,25 \mathrm{~b}$ & $3,50 \mathrm{a}$ & $3,50 \mathrm{a}$ & $3,75 \mathrm{a}$ \\
\hline Conservax & $1,00 \mathrm{a}$ & $1,25 \mathrm{a}$ & $1,50 \mathrm{~b}$ & $1,50 \mathrm{~d}$ & $2,00 \mathrm{ab}$ & $2,75 \mathrm{a}$ & $2,75 \mathrm{a}$ \\
\hline \multirow[t]{2}{*}{$\mathrm{CV}$} & \multicolumn{4}{|c|}{$30,52 \%$} & \multicolumn{3}{|c|}{$29,30 \%$} \\
\hline & \multicolumn{7}{|c|}{ Firmeza $^{2}$} \\
\hline Controle & $1,75 \mathrm{a}$ & $2,25 \mathrm{a}$ & $3,50 \mathrm{a}$ & $4,50 \mathrm{a}$ & $4,00 \mathrm{a}$ & $4,00 \mathrm{a}$ & $3,75 \mathrm{ab}$ \\
\hline PEBD & $2,00 \mathrm{a}$ & $2,00 \mathrm{a}$ & $2,00 \mathrm{~b}$ & $2,50 \mathrm{~b}$ & $3,75 \mathrm{a}$ & $4,00 \mathrm{a}$ & $4,00 \mathrm{a}$ \\
\hline PEBD + sachê & $2,00 \mathrm{a}$ & $2,00 \mathrm{a}$ & $2,50 \mathrm{~b}$ & $2,50 \mathrm{~b}$ & $3,50 \mathrm{a}$ & $3,50 \mathrm{a}$ & $3,50 \mathrm{ab}$ \\
\hline PVC & $1,75 \mathrm{a}$ & $2,00 \mathrm{a}$ & $2,50 \mathrm{~b}$ & $2,50 \mathrm{~b}$ & $4,00 \mathrm{a}$ & $4,00 \mathrm{a}$ & $4,00 \mathrm{a}$ \\
\hline Conservax & $2,00 \mathrm{a}$ & $2,25 \mathrm{a}$ & $2,00 \mathrm{~b}$ & $2,00 \mathrm{~b}$ & $3,25 \mathrm{a}$ & $3,25 \mathrm{a}$ & $3,00 \mathrm{~b}$ \\
\hline $\mathrm{CV}$ & \multicolumn{4}{|c|}{$17,61 \%$} & \multicolumn{3}{|c|}{$11,40 \%$} \\
\hline
\end{tabular}

Para análise estatística os dados foram transformados em $\sqrt{x}$.

Médias seguidas pela mesma letra na coluna não diferem entre si pelo teste Tukey a $5 \%$.

${ }^{1}$ Desenvolvimento de manchas: 1 -fruto sadio, sem manchas; 2 -fruto com pequenas manchas ( 1 a $2 \mathrm{~mm}$ ) e em pequena quantidade. Fruto próprio para consumo, sem restrições; 3-fruto com pequenas manchas, em maior intensidade, ou poucas manchas maiores. Fruto próprio para o comércio, porém com restrições; 4-fruto com $1 / 3$ ou mais de sua superfície tomada por manchas. Fruto sem condições de aproveitamento e 5-Fruto com 2/3 ou mais de sua superfície tomada por manchas. Fruto apodrecido.

${ }^{2}$ Firmeza: 1-muito firme, 2-firme, 3-moderadamente mole, 4-mole e 5-muito mole.

Somente após 21 dias de refrigeração percebeu-se diferença estatística quanto à firmeza, entre o controle e as outras embalagens, sendo que os frutos dos outros tratamentos conseguiram manter melhor a firmeza do que os frutos do controle (Tabela 3). Alves et al. (1998) observaram fato semelhante trabalhando com mangas Tommy Atkins embaladas em PEBD, o qual propiciou melhor preservação da firmeza quando comparado ao controle. Nesta característica, a diferença numérica do PEBD+sachê para o controle é de 1,0 ponto aos 21 dias e 2,0 pontos aos 28 dias (Tabela 3). Com 21 dias em refrigeração e após a permanência de 4 dias em temperatura ambiente, os frutos dos tratamentos PEBD+sachê e Conservax estavam moderadamente moles, enquanto os dos tratamentos controle, PVC e PEBD, apresentavam-se moles.

Não houve influência definida dos tratamentos sobre as características químicas das mangas. Os teores de sólidos solúveis dos tratamentos não apresentaram tendência de aumento durante o armazenamento em refrigeração ou após transferência para temperatura ambiente. Os teores de sólidos solúveis se situaram entre 15,4 e 16,0 Brix, valor este concordante ao alcançado em mangas da variedade Espada, descrito no livro organizado por Donadio (1996). As mangas apresentaram valores crescentes de $\mathrm{pH}$ de 4,2 na colheita a 4,9 referentes aos períodos após 28 dias em refrigeração e após transferências e permanência dos frutos por 4 dias em temperatura ambiente. A acidez total dos frutos decresceu de $0,46 \% \mathrm{em}$ média no ponto de colheita para $0,23 \%$ tanto nas mangas após 28 dias de refrigeração como após permanência de 4 dias em temperatura ambiente. A relação Brix/acidez, que é usada como indicador de palatabilidade de frutos, aumentou de 37,6 na colheita para 57,6 e 67,8 no período final de avaliação, para os frutos mantidos apenas em refrigeração e para os transferidos para temperatura ambiente, respectivamente.

\section{CONCLUSÕES}

1) A embalagem PEBD+sachê teve influência positiva no con- 
trole do aparecimento de manchas deteriorativas e na manutenção da qualidade das mangas refrigeradas por 14 dias seguidos de 4 dias em temperatura ambiente ou refrigeradas por 28 dias e consumo imediato.

2) A embalagem Conservax prejudicou a maturação dos frutos, ficando estes com a casca escurecida, o que provavelmente prejudicaria a sua comercialização.

\section{REFERÊNCIAS BIBLIOGRÁFICAS}

AGRIANUAL 2003. Manga. São Paulo: FNP Consultoria \& Agroinformativos, 2002. p. 392-398.

ALVES, R.M.V.; SIGRIST, J.M.M.; PADULA, M. Atmosfera modificada em mangas "Tommy Atkins". Revista Brasileira de Fruticultura, Cruz das Almas, v.20, n.2, p.220-228, 1998.

CARVALHO, C.R.L; MANTOVANI, D.M.B.; CARVALHO, P.R.N.; MORAES, R.M. Análises químicas de alimentos. Campinas: ITAL, 1990. 121p.

DONADIO, L. C. (Org.) Variedades brasileiras de mangas. São Paulo: Fundação Editora da UNESP, 1996. 74p.

EMEX,A.C. Norma de calidad para mango fresco de exportacion. Mexico: Centro de Investigación en Alimentación y Desarrollo, 1998.4p.

INSTITUTO BRASILEIRO DE FRUTAS. Mercanotas. Informativo IBRAF, São Paulo, n.18, 2003.

JERÔNIMO, E.M. Efeito do uso de embalagens associadas a armazenamento sob refrigeração na conservação pós-colheita de mangas 'Tommy Atkins' e 'Palmer'. 2000. 121f. Dissertação (Mestrado em Produção Vegetal) - Faculdade de Ciências Agrárias e
Veterinárias, Universidade Estadual Paulista. Jaboticabal - SP, 2000. JERÔNIMO, E.M.; KANESIRO, M.A.B. Efeito da associação de armazenamento sob refrigeração e atmosfera modificada na qualidade de mangas 'Palmer'. Revista Brasileira de Fruticultura, Jaboticabal, v.22, n.2, p.237-243, 2000.

LANA, M.M.; FINGER, F.L. Atmosfera modificada e controlada: aplicação na conservação de produtos hortícolas. Brasília: Embrapa Comunicação para Transferencia de Tecnologia, 2000. 34 p.

MEDLICOTT, A.P.; SIGRIST, J.M.M.; SY, O. Ripening of mangoes following low temperature storage. Journal of the American Society for Horticultural Science, Alexandria, v.115, n.2, p.430-434, 1990.

POLDERDIJK, J.J.; KHO, R.M.; KRUIF, A.P.M.; CARMI, Y. Firmness of mangoes (Mangifera indica L.) measured acoustically, mechanically and manually. Acta Horticulturae, Wageningen, v.509, p.861-865, 2000.

ROSSETTO, C.J.; GALLO, P.B.; BORTOLETTO, N.; CARVALHO, C.R.L.; RIBEIRO, I.J.A.; CASTRO, J.V. Manga IAC Espada Vermelha. In: DONADIO, L. C. (Ed.) Novas variedades brasileiras de frutas. Jaboticabal: Sociedade Brasileira de Fruticultura, 2000. p.138-139.

SAMPAIO, V.R.; BARBIN, D. Controle em pós-colheita das podridões de frutos de manga conservados na câmara fria. In: CONGRESSO BRASILEIRO DE FRUTICULTURA, 6., 1981, Recife. Anais... Recife: Sociedade Brasileira de Fruticultura, 1981. p.952-962.

SARANTÓPOULOS, C.I.G.L.;ALVES, R.M.V.A.; OLIVEIRA, L.M. Embalagens com atmosfera modificada. Campinas: CETEA: ITAL, 1996. $114 \mathrm{p}$. 